\title{
Nanotomography of Inverse Photonic Crystals Using Zernike Phase Contrast
}

$\underline{\text { Silja Flenner }}^{1}$, Emanuel Larsson ${ }^{1}$, Kaline Furlan ${ }^{2,3}$, Daniel Laipple ${ }^{1}$, Malte Storm ${ }^{4}$, Fabian Wilde ${ }^{1}$, Robert Blick $^{3}$, Gerold A. Schneider ${ }^{2}$, Robert Zierold ${ }^{3}$, Rolf Janssen ${ }^{2}$, Christian David ${ }^{5}$, Felix Beckmann ${ }^{1}$, Martin Müller ${ }^{1}$ and Imke Greving ${ }^{1}$

1. Institute of Materials Research, Helmholtz-Zentrum Geesthacht, Geesthacht, Germany.

2. Institute of Advanced Ceramics, Hamburg University of Technology (TUHH), Hamburg, Germany.

3. Center for Hybrid Nanostructures, Universität Hamburg, Hamburg, Germany.

4. Diamond Light Source, Didcot, UK.

${ }^{5 .}$ Paul Scherrer Institut, Villigen, Switzerland.

* Corresponding author, silja.flenner@hzg.de

$\mathrm{X}$-ray full field microscopy using synchrotron radiation is a frequently used tool in material science. In particular for low absorbing samples, however, the contrast is often not sufficient for tomography. To enhance the contrast of this type of materials the established technique of Zernike Phase Contrast (ZPC) $[1,2,3]$ has been implemented at the Nanotomography endstation of the Imaging Beamline P05 at PETRA III storage ring, located at DESY (Hamburg, Germany). Using an energy of $11 \mathrm{keV}$, a spatial resolution of $100 \mathrm{~nm}$ was achieved and the obtained high contrast made 3D investigation of low absorbing materials possible. Inverse photonic crystals consisting of hollow spheres absorb very little due to their high air content and are therefore an ideal test system for the ZPC tomography setup.

A schematic layout of a Zernike Phase contrast setup, as implemented at the P05 Nanotomography endstation, is shown in Figure 1. The beam shaper collects the incoming, monochromized synchrotron $\mathrm{X}$-ray beam and creates the required hollow cone illumination. A circular beam shaper with a diameter of $0.9 \mathrm{~mm}$ and subfields of $50 \mu \mathrm{m}$ was implemented. The central stop was chosen to be $0.2 \mathrm{~mm}$ in diameter and blocks the central beam. The nanotomography setup is equipped with a high precision airbearing rotation axis. A six axis kinematics is located in the center, allowing for centering of the sample with respect to the rotation axis. A Fresnel zone plate (FZP) with an outer zone width of $50 \mathrm{~nm}$ is placed at a focal distance of $84 \mathrm{~mm}$ and magnifies the image onto the detector. The detector (Hamamatsu, $6.5 \mu \mathrm{m}$ pixel size, $10 \mu \mathrm{m}$ Gadox Scintillator) is placed in the next experimental hutch at $18.8 \mathrm{~m}$ sampleto-detector distance, resulting in an effective pixel size of $28.7 \mathrm{~nm}$. Thanks to this large distance, no light optical magnification is necessary, which increases the photon efficiency significantly. The phase rings for ZPC is placed in the back focal plane of the objective lens, which creates a phase shift of the non-diffracted beam. All optics were fabricated at the Paul Scherrer Institut (Switzerland).

For first 2D tests, a Siemens Star test pattern with $25 \mathrm{~nm}$ smallest feature size was used. Figure 2 shows flat field corrected projections of the test sample without (a) and with ZPC (b). The ZPC images show a much better contrast and a better signal-to-noise ratio compared to the absorption contrast image (a).

A first tomographic scan using this ZPC setup was performed on an inverse photonic crystal (i-PhC), which has a highly porous periodically ordered structure and is produced by Atomic Layer Deposition (ALD). Due to this 3D periodic structure, these materials are capable of affecting the propagation of electromagnetic radiation (photonic band gap). In particular for high-temperature applications, such as next-generation thermal barrier coatings (photonic TBCs) [4] or thermo photovoltaic energy conversion devices [5] this material is of relevant interest. Therefore, the effect of heat treatments on the inner 
structure of this material is of major importance. Understanding the structure destabilization mechanisms will allow for an optimization of the synthesis process of the i-PhC. In order to fully characterize the three-dimensional structure of the i-PhC a tomographic scan was performed at the imaging beamline P05 using ZPC. The reconstructed slice of this i-PhC sample (Figure 3) reveals a drastic change of the periodic structure after an extensive heat treatment at $140{ }^{\circ} \mathrm{C}$ for 100 hours, which results in loss of reflectance capability.

The first results of ZPC performed at P05 show that this is an ideal technique to study the inner structure of low absorbing materials like inverse photonic crystals, without the need of staining. In comparison to standard absorption contrast methods, we obtained much better contrast and heat induced structural defects of inverse photonic crystals could be revealed.

\section{References:}

[1] F. Zernike, Physica 1, (1934) p. 689-704.

[2] G. Schmahl et al, Optik 97 (1994) p. 181-182.

[3] I. Vartiainen et al, Journal of Synchrotron Radiation 21 (2014) p. 790-794.

[4] H.S. Lee et al, Journal of the Optical Society of America B-Optical Physics 29 (3) (2012) p. 450.

[5] Y.X. Yeng et al, Proc. Natl. Acad. Sci. USA 109 (7) (2012) p. 2280-2285.

[6] The authors SF, EL, KF, GS, MM, IG acknowledge financial support from the German Research Foundation (DFG) via the SFB 986-M3.

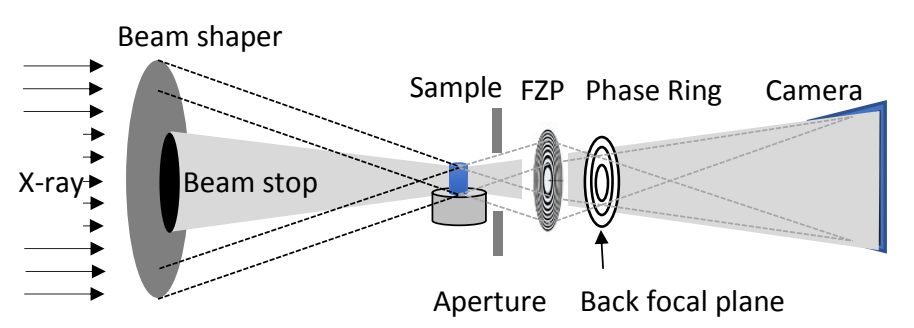

Figure. 1. Schematic of the ZPC setup at the imaging beamline P05.

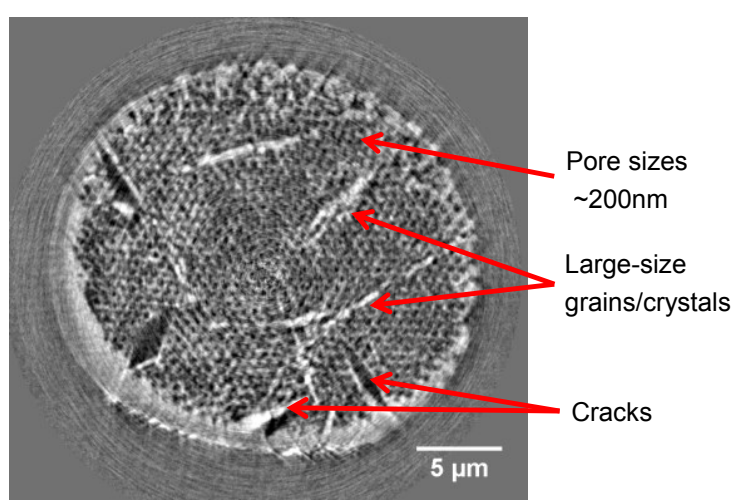

Figure. 3. Reconstructed slice of an inverse photonic crystal. The initial macro pore size of $762 \mathrm{~nm}$ is drastically reduced down to $\sim 200 \mathrm{~nm}$ and cracks as well as large crystals are revealed.
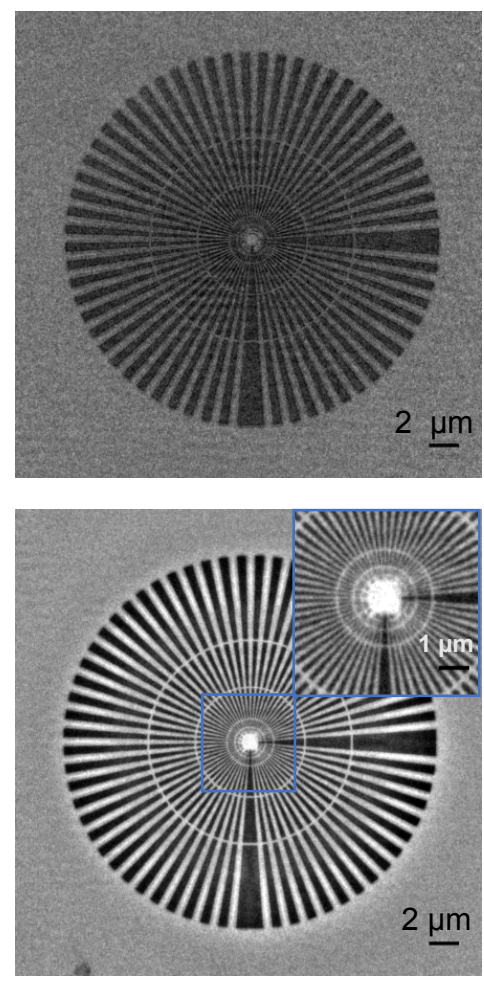

Figure. 2. Different contrast methods of the test sample (flatfield corrected projections, no filters applied). a) Absorption contrast, b) Zernike phase contrast. 